Retrospective Study

\title{
The Clinical Results of Percutaneous Endoscopic Interlaminar Discectomy (PEID) in the Treatment of Calcified Lumbar Disc Herniation: A Case-Control Study
}

Xu Dabo, MS ${ }^{1,2}$, Ziqiang Chen, MD¹, Zhao Yinchuan, MD1, Ni Haijian, MD1, Chen Kai, MD1, Liu Yanbin, MS'1, Fu Qiang, MD¹, and Wang Chuanfeng, MD ${ }^{3}$

From: ${ }^{1}$ Department of Spine Surgery, Changhai Hospital, the Second Military Medical University, Shanghai, China; 2Department of Orthopedics, Ningbo No.7 Hospital, Ningbo, Zhejiang, China; ${ }^{3}$ Department of Orthopedics, Changhai Hospital, the Second Military Medical University, Shanghai, China

Address Correspondence: Fu Qiang,MD Department of Spine Surgery, Changhai Hospital, the Second Military Medical University, Shanghai, China E-mail: johson.f@163.com Wang Chuanfeng,MD Department of Orthopedics, Changhai Hospital,the Second Military Medical

University, Shanghai, China E-mail: cfeng_wong@163.com

Disclaimer: Fu Qiang and Wang Chuanfeng contributed equally to this work. There was no external funding in the preparation of this manuscript. Conflict of interest: Each author certifies that he or she, or a member of his or her immediate

family, has no commercial association (i.e., consultancies, stock ownership, equity interest, patent/licensing arrangements, etc.) that might pose a conflict of interest in connection with the submitted manuscript.

Manuscript received: 12-04-2014 Revised manuscript received 04-28-2015:

Accepted for publication: 09-02-2015

Free full manuscript: www.painphysicianjournal.com
Background: Percutaneous endoscopic interlaminar discectomy (PEID), which poses advantages for certain types of herniated disc, is gaining wider acceptance in clinical practice. We retrospectively analyzed the efficacy of the PEID technique in treatment of calcified lumbar disc herniation.

Study Design: A retrospective case-control study.

Setting: University hospital in China.

Objective: To evaluate the efficacy of the PEID technique in treatment of calcified lumbar disc herniation, and a comparison between calcified and noncalcified disc herniation was drawn to analyze the causes of herniated disc calcification.

Methods: Data from patients who underwent full-endoscopic lumbar discectomy in our department between March 2011 and May 2013 were collected. Thirty cases with calcified lumbar disc herniation were included in the study group, and 30 age-, gender-, and body mass index (BMI)-matched cases with noncalcified lumbar disc herniation served as controls. Perioperative data, preoperative and postoperative Visual Analog Scale (VAS) scores, Oswestry Disability Index (ODI) values, MacNab scores, and postoperative low-extremity dysesthesia among patients in the 2 groups were collected.

Results: The values of computed tomography $(\mathrm{CT})$ in the calcified group were significantly higher than those in the noncalcified group $(P<0.01)$. The preoperative disease courses in the 2 groups were similar. However, there was a statistically significant difference in the duration of traditional Chinese medicines (TCM) administration $(P<0.01)$. VAS and ODI scores improved significantly after surgery, but there were no significant differences between the 2 groups $(P>$ $0.05)$. Three months after surgery, the rate of low-extremity dysesthesia in the calcified group was significantly higher than that in the control group $(P=0.03)$ but became similar at 6 months. By applying MacNab criteria the proportions of good and excellent were greater than $90 \%$ in both groups, and there was no difference between groups $(P>0.05)$.

Limitations: The sample size was small in this retrospective study.

Conclusion: The PEID technique is an effective method in the treatment of calcified lumber disc herniation, although the rate of postoperative dysesthesia is higher in this group during the early postoperative period. Long-term TCM administration may be related to the calcification of herniated lumbar discs.

Key words: Lumbar disc herniation, percutaneous endoscopic lumbar discectomy, interlaminar approach, calcification

Pain Physician 2016; 19:69-75 
alcified lumbar disc herniation is a special type of lumbar disc herniation. Specific reasons for The formation of calcification are not yet clear. Patients with calcified lumbar disc herniation usually have a long course of conservative treatment prior to consideration of surgery. Jasper et al (1) considered that mineral deposit formation and even calcification might occur in the herniated nucleus pulposus when the disease course of lumbar disc herniation exceeded 6 months, which makes surgical treatment difficult compared with treatment during the early stages of the disease. Nowadays, literature about cases with calcified lumbar disc herniation are rare except in China. Calcified lumbar disc herniation has become a challenging problem for spine surgeons in China. Is this related to the different types of conservative treatments? In China, conservative treatments include not only lumbar traction and other physiotherapies but also administration of Traditional Chinese Medicines (TCM).

Microdiscectomy was not recommended for calcified disc herniation as the risk increased significantly if the calcified herniated disc was treated by the transforaminal approach $(2,3)$. In addition, the risk of nerve root injury is elevated during traditional open surgery for calcified disc herniation. Therefore, Choi et al (4) removed a calcified disc herniation in the upper lumbar spine via a transdural approach. However, this approach may induce cerebrospinal fluid leakage and central nervous system infection.

With the improvement of surgical techniques and related instruments, indications for minimally invasive surgery have expanded and include revision surgery for recurrent lumbar disc herniation and lumbar spinal stenosis (5-9). However, only targeted direct decompression within the spinal canal can completely release the nerve root in cases with calcified disc herniation. Percutaneous endoscopic interlaminar discectomy (PEID), which avoids factors such as a high iliac crest and transverse process variation, is a surgical procedure that can directly remove the lesion inside the spinal canal and has obvious advantages in the treatment of some special types of disc herniation such as hard disc herniation and craniocandal sequestering of the disc marterial (10-14). PEID can be applied for minimally invasive treatment of calcified disc herniation. We retrospectively analyzed and compared the efficacy of PEID in the treatment of calcified and noncalcified disc herniation. In addition, in this study, we found that the TCM treatment may have certain relevance with calcifi- cation of intervertebral disc herniation lesions, and we further analyzed the etiology.

\section{Methods}

\section{General Data}

At present, there are no specific diagnostic criteria for calcified lumbar disc herniation. Among patients with a definite diagnosis of lumbar disc herniation, we selected those who had a computed tomography (CT) value of the herniated disc close to the bone (120.1383.7 Hu) and defined this kind of situation as calcified lumbar disc herniation. All patients had a central or paracentral disc herniation, and there was no case with far lateral disc herniation. Exclusion criteria included the following: (1) multisegmental lumbar disc herniations; (2) extensive spinal canal stenosis without disc herniation in CT or magnetic resonance imaging (MRI); sequestrated disc fragments exceeding more than half of the upper or lower vertebral body; less than $6 \mathrm{~mm}$ of distance between the posterior midline and the inner margin of the inferior facet joint (10); (3) patients with sudden and progressive aggravating painless weakness and cauda equina syndrome.

Among patients who underwent PEID in our department from March 2011 to May 2013, we selected 30 patients who had calcified lumbar disc herniation (CLDH group) in accordance with the standard mentioned above, chose 30 cases with noncalcified disc herniation (NCLDH group) as the control group, and matched the controls in terms of gender, age, and body mass index (BMI). The general clinical data of the 2 groups were collected. Clinic records were reviewed to document their surgical results. Preoperative and follow-up data were obtained from clinic visits, physical and occupational therapy records, and telephone interviews. Preoperative disease course and usage of TCM (total administrating time of TCM and continuous administrating time > 3 months per person) were recorded. Among them only the usage of phenol-rich herbal patches and oral pills of patent Chinese drugs were recorded as an entire list of formulas and ingredients of TCM was impossible.

\section{Surgical Procedure}

The full endoscopic surgical system (Joimax $\mathrm{GmbH}$, Karlsruhe, Germany) was used. General anesthesia was applied for all patients. The surgical procedure was performed by 2 surgeons. The diseased segment was located under fluoroscopic guidance, and the needle insertion point was marked. An incision 
$7 \mathrm{~mm}$ in length was made, and a dilator was inserted under fluoroscopy to reach the intervertebral space. The sheath and endoscope were inserted in turn, and the irrigation system was connected. The sheath was rotated to push aside the peripheral muscles. The ligamentum flavum was cut off using a pituitary rongeur. The dura mater was exposed after removal of the fat tissue. For a calcified central or axillary herniation, the nerve root was identified using a nerve dissector along the lateral recess under endoscopy. Because the nerve root travels horizontally at the L5/S1 level, it is safe to manage the axillary lesion first. The oblique distal opening of the sheath should be toward the midline of the spinal canal to effectively expose the lesion and protect the nerve root and dura mater. After hemostasis, the pannus and adhesion bands were removed, the calcified lesion was removed using a trephine under endoscopy, and the cottony tissues produced by incomplete calcification were also handled. It is not necessary to remove all the calcified lesion, and the intervertebral structure should not be disturbed excessively. The rupture in the annulus fibrosus was managed using radiofrequency current. All patients started straight leg raise exercise and ambulated suitably on the first day after surgery, then the patients exercised their back muscles increasingly.

\section{Outcome Observations}

A visual analog scale (VAS, 0 - 10) was used for preoperative and postoperative leg pain evaluation. The Oswestry Disability Index (ODI, version 2.1a) was used to assess the influence of back pain and leg pain on the daily lives of patients (15). Because sex is not routinely discussed among Chinese people, the item regarding sexual life was deleted. Therefore, there were 9 items left, and the total possible score was 45 points. Scores of every item were added, and the percentages of these scores were calculated. The presence of postoperative lower-extremity dysesthesia was recorded at various times after surgery in both groups. The final outcome was evaluated according to MacNab's criteria (16).

\section{Statistical Methods}

Data analysis was performed using SPSS version 20.0 (SPSS Inc., Chicago, IL, USA). Continuous data were compared between the CLDH and NCLDH groups, using the independent Student t test or the Mann-Whitney $U$ test, whereas discontinuous data were analyzed using Fisher's exact test, the chi-square test. All significance tests were 2-tailed, and a positive significance level was assumed at a probability of $P<0.05$.

\section{Results}

A typical case in the calcified group is shown in Fig. 1. The general conditions of patients in the 2 groups are shown in Table 1. There were no significant differ-
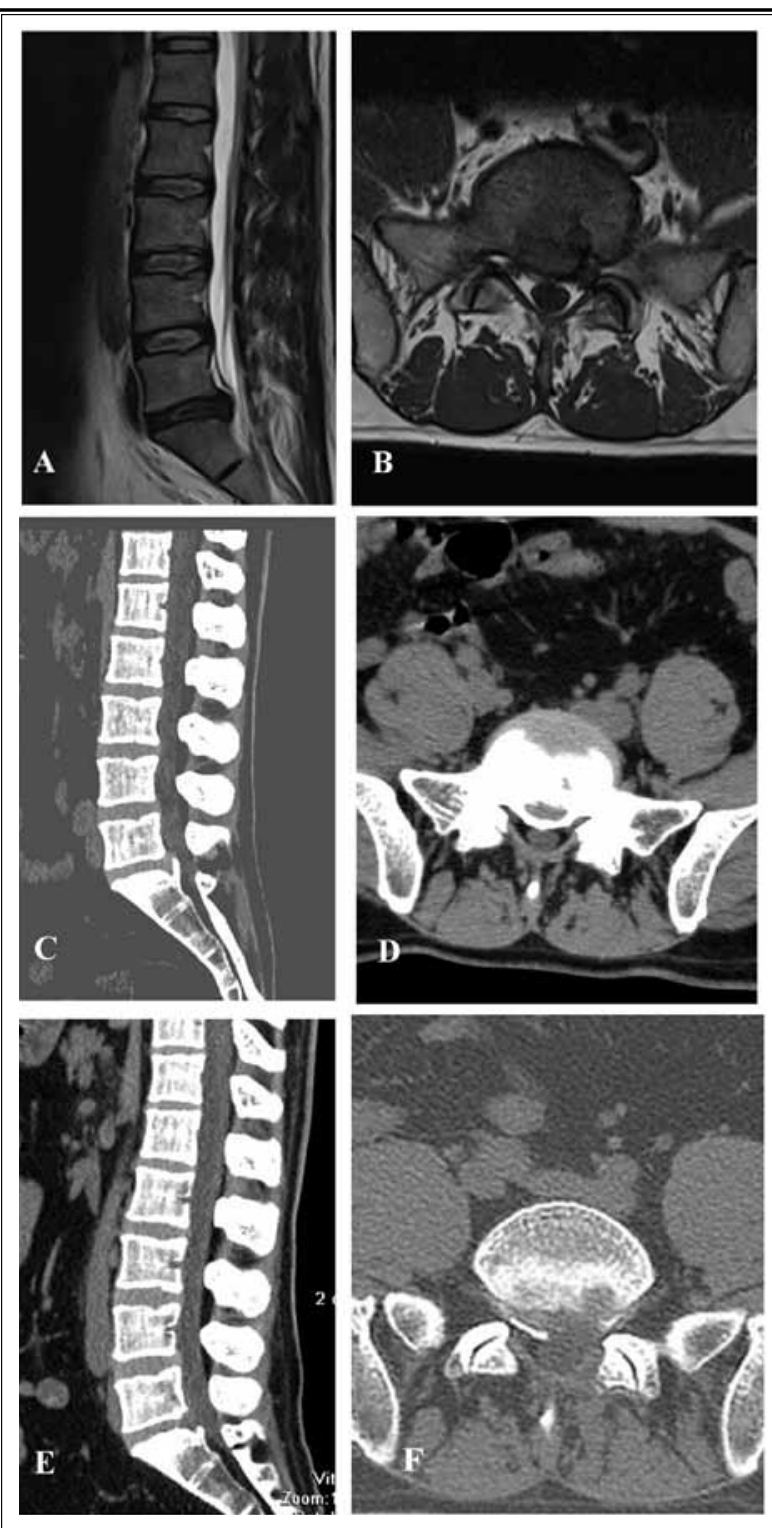

Fig. 1. A 38-year-old man presented with lower back pain radiating to the left leg for $>2$ years. The patient was diagnosed with L5/S1 disc herniation (left central) and underwent fullendoscopic interlaminar discectomy. Preoperative MRI and three-dimensional CT showed calcified L5/S1 disc herniation ( $A, B, C$, and D). Three-dimensional CT obtained 3 months after surgery showed that the calcified lesion around the left $S 1$ nerve root was completely removed ( $E$ and $F$ ). 
Table 1. Comparison of perioperative general conditions in both groups.

\begin{tabular}{|c|c|c|c|c|}
\hline & & $\begin{array}{c}\text { CLDH } \\
(\mathbf{n}=\mathbf{3 0})\end{array}$ & $\begin{array}{l}\text { NCLDH } \\
(n=30)\end{array}$ & $P$-value \\
\hline \multicolumn{2}{|l|}{ Age(y) } & $36.0 \pm 7.8$ & $35.9 \pm 6.5$ & 0.957 \\
\hline \multirow{2}{*}{ Sex } & M & 20 & 18 & \multirow{2}{*}{$0.592 \#$} \\
\hline & $\mathrm{F}$ & 10 & 12 & \\
\hline \multicolumn{2}{|l|}{ BMI } & $26.1 \pm 3.1$ & $25.2 \pm 2.9$ & 0.248 \\
\hline \multirow{2}{*}{ Segments } & $\mathrm{L} 4 / 5$ & 12 & 9 & \multirow{2}{*}{$0.417 \#$} \\
\hline & L5/S1 & 18 & 21 & \\
\hline \multicolumn{2}{|c|}{ CT values of herniated disc (HU) } & $288.8 \pm 63.6$ & $56.0 \pm 17.8$ & $<0.01^{*}$ \\
\hline \multicolumn{2}{|c|}{ Length of stay (d) } & $5.9 \pm 1.1$ & $5.9 \pm 1.0$ & 0.90 \\
\hline \multicolumn{2}{|c|}{ Operative time (min) } & $57.6 \pm 11.2$ & $50.6 \pm 8.7$ & $<0.01^{*}$ \\
\hline
\end{tabular}

\#Chi-square test, ${ }^{\star}$ Mann-Whitney test; a P value $<0.05$ was considered to be statistically significant.

ences in gender, age, BMI, disease segment, and length of stay $(P>0.05)$. There was a significant difference between the 2 groups in the CT value of the herniated $\operatorname{disc}(288.8 \pm 63.6$ vs. $56.0 \pm 17.8, P<0.01)$. The operative time in the CLDH group was significantly longer than that in the NCLDH group $(P<0.01)$.

The preoperative disease course and administration of TCM were recorded in Table 2. There was no significant difference in the preoperative disease course $(P=0.235)$. However, there was a significant difference between the 2 groups regarding the total administrating time of TCM $(7.9 \pm 4.3$ vs. $3.7 \pm 2.4, P<0.01)$, and the number of patients who had undergone continuous TCM treatment $>3$ months was significantly larger than that in the control group (24 vs. $13, P<0.01$ ).

As shown in Fig. 2, there were significant differences in preoperative and postoperative VAS and ODI scores in both groups $(P<0.01)$; there was no significant difference between various points of time in the VAS score for leg pain in both groups $(P>0.05)$, but the VAS score of the NCLDH group was slightly better than that of the CLDH group in the early postoperative period $(P>0.05)$. The ODI scores at various points of time in the NCLDH group were slightly better than those in the CLDH group, but there was no significant difference between the 2 groups at the last follow-up visit $(P>0.05)$.

Three months after surgery, 16 patients in the CLDH group and 8 patients in the NCLDH group had varying degrees of postoperative dysesthesia, and the difference was significant $(P=0.03)$. Six months after surgery, 9 patients in the CLDH group and 4 patients in the NCLDH group had remnant lower-extremity dysesthesia, but the difference between the 2 groups was not significant $(P=0.209)$. Twelve months after surgery, 3 patients in the CLDH group and 2 patients in the NCLDH group had lower-extremity dysesthesia ( $P$ $=1.00)$. Patients in both groups did not have any complications such as nerve root laceration, cauda equina syndrome, wound infection, spinal canal hematoma, incision infection, or infection of the central nervous system. One patients had a dural laceration in the CLDH group, but no special intraoperative treatment was carried out for the small laceration, and no other postoperative complications appeared. There are no recurrences of lumbar disc herniation in any patients at the end of follow-up.

MacNab's criteria results were excellent in 24 cases, good in 3 cases, fair in 2 cases, and poor in one case in the CLDH group; excellent in 27 cases, good in 2 cases, and fair in one case in the NCLDH group. The percentage of results rated good to excellent were $90 \%$ and $96.7 \%$ in the CLDH group and the NCLDH group at the final follow-up visit, respectively, and there was no significant between group difference $(P=0.635)$.

\section{Discussion}

Recently, Ruetten et al $(10)$ and Choi et al $(11,17,18)$ reported a full-endoscopic interlaminar technique in which the surgeon can reach the spinal canal via the interlaminar space to remove the herniated central and paracentral lesions, and this technique is effective for patients with highly migrated nucleus pulposus or discogenic stenosis. Choi et al (11) described in detail the anatomical features of the S1 nerve root at the L5/ S1 level. They found many axillary-type disc herniations and suggested that the interlaminar approach was safe for reaching the axillary space. When surgeons remove 
Table 2. Preoperative disease course and administration of TCM.

\begin{tabular}{|c|c|c|c|c|}
\hline & & $\begin{array}{c}\text { CLDH } \\
(n=30)\end{array}$ & $\begin{array}{l}\text { NCLDH } \\
(n=30)\end{array}$ & $P$-value \\
\hline \multicolumn{2}{|l|}{ Preoperative disease course (m) } & $24.7 \pm 10.9$ & $21.0 \pm 12.7$ & 0.235 \\
\hline \multicolumn{2}{|c|}{ Total administrating time of TCM $(\mathrm{m})$} & $7.9 \pm 4.3$ & $3.7 \pm 2.4$ & $<0.01^{*}$ \\
\hline \multirow{2}{*}{ Continuous TCM treatment } & $>3 \mathrm{~m}$ & 24 & 13 & \multirow{2}{*}{$<0.01 \#$} \\
\hline & $<3 \mathrm{~m}$ & 6 & 17 & \\
\hline
\end{tabular}

${ }^{*}$ Mann-Whitney test; \#Chi-square test, a P value $<0.05$ was considered to be statistically significant.

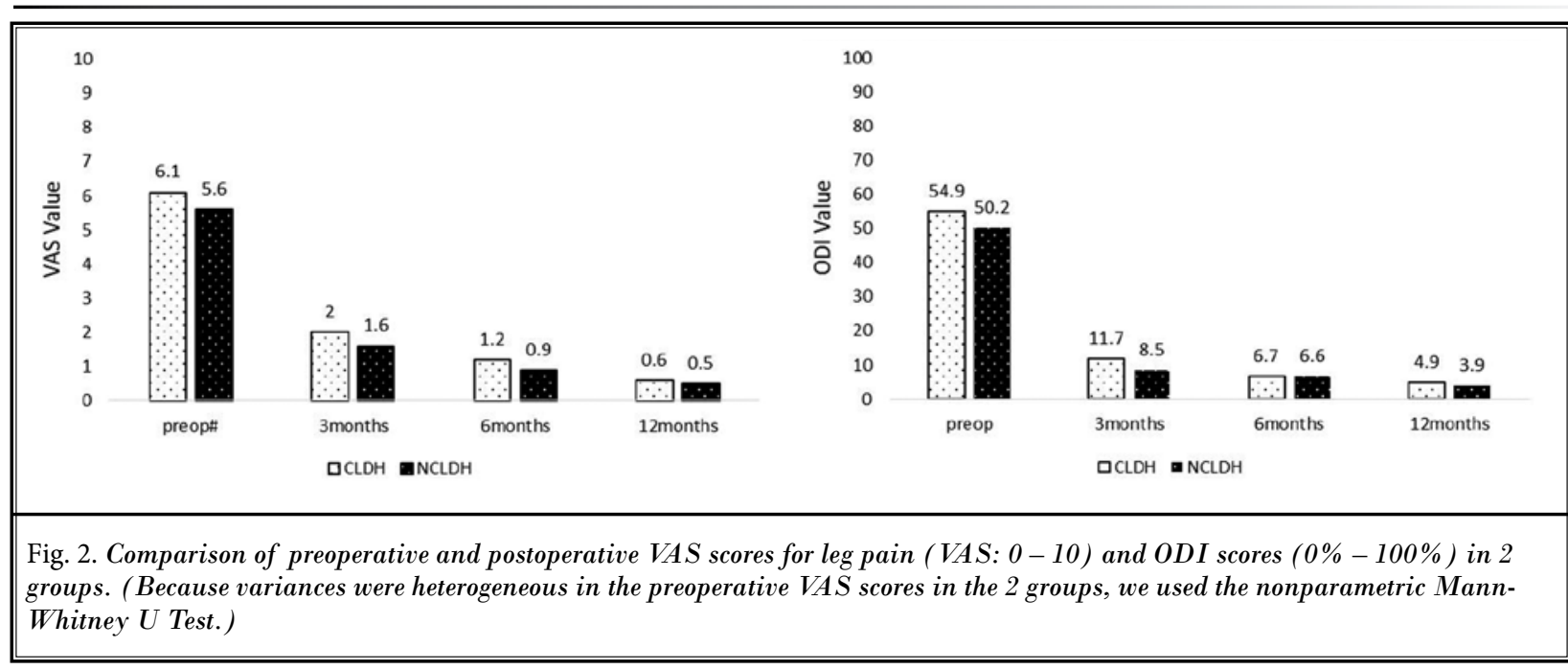

the shoulder-type disc herniation, they should remove the medial part of the inferior articular process to expand the interlaminar space for operation $(19,20)$. For experienced surgeons who are familiar with the application of endoscopic instruments such as the trephine and laser, the interlaminar approach can make the bone resection easier, which expands the operating space to facilitate reaching the lesion directly and thereby reducing surgical complications $(9,21-23)$.

The texture of the calcified disc herniation is hard and the calcified lesion often adheres to the nerve root and dura mater, and the latter tissues are easily injured during blind dissection. In addition, calcified disc herniation is often associated with discogenic spinal stenosis, which complicates transforaminal discectomy (24-28). In the treatment of calcified disc herniation, the interlaminar approach can enable surgeons to directly reach the lesion without obviously disturbing the normal bone structure and enabling relatively safe gradual decompression from the top of the lesion. We believe that the goal for the treatment of calcified disc herniation is not to achieve complete radiographic resection but rather to decompress the nerve root, which agrees with the opinions of many scholars (29-34).

Researchers compared the surgical outcomes of the interlaminar and the transforaminal approaches and found that postoperative lower-extremity dysesthesia occurred mainly during the interlaminar approach $(2,12,19)$. In the present study, we found that the incidence of postoperative dysesthesia was relatively high in the calcified group compared with that in the noncalcified group. Dysesthesia generally occurred during the early postoperative period, and resection of the lesion step-by-step under direct vision can reduce the risk of potential nerve root injury (35). So it is necessary to pay close attention to preoperative patient selection, to carefully measure the size of the related interlaminar space, and to evaluate the relationship between the intervertebral disc and the upper and lower laminae in order to avoid nerve injury. For patients with calcified disc herniation, a preoperative CT scan is needed to assess the range of the calcified lesion (36). Nerve root traction caused by the rotation of the trephine may result in lower-extremity dysesthesia during the early postoperative period.

The technique used in the present study is different 
from transforaminal foraminotomy $(37,38)$. Sufficient attention should be paid to intraoperative protection of the dura mater. In order to prevent cerebrospinal fluid leakage, care should be taken not to tear the dura during rotation. Sufficient posterior longitudinal ligament should be removed and a flexible neural probe was used to dissect peripheral connective tissue before careful removal of the calcified lesion.

In other countries, reports about calcified lumbar disc herniation are rare. However, reports suggest that the incidence of calcified lumbar disc herniation is higher in China than elsewhere (39). Is the high incidence of calcified lumbar disc herniation in China related to long-term TCM administration? Most TCMs can promote blood circulation, take away inflammatory cytokines from the lesion, and reduce pain. However, TCM cannot make the herniated disc disappear.
Although no reports have confirmed the correlation between calcifications and TCM, in the present study we found that the duration of TCM administration in the calcified group was significantly longer than that in the control group. There may be a correlation between TCM administration and calcification of the herniated disc, but of course this should be further verified.

\section{Conclusion}

Though PEID for calcified lumbar disc herniation easily results in residual symptoms such as lowerextremity dysesthesia during the early postoperative period, its final outcome is similar to outcomes in patients with noncalcified lumbar disc herniation. Longterm TCM administration may be one of the causes for calcification of the herniated disc.

\section{References}

1. Jasper GP, Francisco GM, Telfeian AE. Clinical success of transforaminal endoscopic discectomy with foraminotomy: A retrospective evaluation. Clin Neurol Neurosurg 2013; 115:1961-1965.

2. Birkenmaier C, Komp M, Leu HF, Wegener $B$, Ruetten $S$. The current state of endoscopic disc surgery: Review of controlled studies comparing full-endoscopic procedures for disc herniations to standard procedures. Pain Physician 2013; 16:335-344.

3. Dezawa A, Sairyo K. New minimally invasive discectomy technique through the interlaminar space using a percutaneous endoscope. Asian J Endosc Surg 2011; 4:94-98.

4. Choi JW, Lee JK, Moon KS, Hur H, Kim YS, Kim SH. Transdural approach for calcified central disc herniations of the upper lumbar spine. Technical note. J Neurosurg Spine 2007; 7:370-374.

5. Kubaszewski L, Kaczmarczyk J, Nowakowski A, Sulewski A. Foraminoplastic transfacet epidural endoscopic approach for removal of intraforaminal disc herniation at the $\mathrm{L}_{5}$-S1 level. Wideochir Inne Tech Malo Inwazyjne 2014; 9:96-100.

6. Jasper GP, Francisco GM, Aghion D, Telfeian AE. Technical considerations in transforaminal endoscopic discectomy with foraminoplasty for the treatment of spondylolisthesis: Case report. Clin Neurol Neurosurg 2014; 119:84-87.

7. Ruetten S, Komp M, Hahn P, Oezdemir $\mathrm{S}$. Decompression of lumbar lateral spi- nal stenosis: Full-endoscopic, interlaminar technique. Oper Orthop Traumatol 2013; 25:31-46.

8. Ruetten S, Komp M, Merk H, Godolias G. Surgical treatment for lumbar lateral recess stenosis with the full-endoscopic interlaminar approach versus conventional microsurgical technique: A prospective, randomized, controlled study. J Neurosurg Spine 2009; 10:476-485.

9. Komp M, Hahn P, Merk H, Godolias G, Ruetten S. Bilateral operation of lumbar degenerative central spinal stenosis in full-endoscopic interlaminar technique with unilateral approach: Prospective 2-year results of 74 patients. J Spinal Disord Tech 2011; 24:281-287.

10. Ruetten S, Komp M, Godolias G.A New full-endoscopic technique for the interlaminar operation of lumbar disc herniations using 6-mm endoscopes: Prospective 2-year results of 331 patients. Minim Invasive Neurosurg 2006; 49:80-87.

11. Choi G, Lee SH, Raiturker PP, Lee S, Chae YS. Percutaneous endoscopic interlaminar discectomy for intracanalicular disc herniations at $\mathrm{L}_{5}$-Si using a rigid working channel endoscope. Neurosurgery 2006; 58:ONS59-ONS68; discussion ONS59-ONS68.

12. Ruetten S, Komp M, Merk H, Godolias G. Use of newly developed instruments and endoscopes: Full-endoscopic resection of lumbar disc herniations via the interlaminar and lateral transforaminal approach. J Neurosurg Spine 2007;
6:521-530.

13. Ruetten S, Komp M, Merk H, Godolias G. Recurrent lumbar disc herniation after conventional discectomy: A prospective, randomized study comparing full-endoscopic interlaminar and transforaminal versus microsurgical revision. J Spinal Disord Tech 2009; 22:122-129.

14. Kim CH, Chung CK, Jahng TA, Yang HJ, Son YJ. Surgical outcome of percutaneous endoscopic interlaminar lumbar diskectomy for recurrent disk herniation after open diskectomy. J Spinal Disord Tech 2012; 25:E125-E133.

15. Liu $\mathrm{H}$, Tao $\mathrm{H}$, Luo Z. Validation of the simplified Chinese version of the Oswestry Disability Index. Spine (Phila $\mathrm{Pa}$ 1976) 2009; 34:1211-6; discussion 1217.

16. MacNab I. Negative disc exploration. An analysis of the causes of nerve-root involvement in sixty-eight patients. J Bone Joint Surg Am 1971; 53:891-903.

17. Choi G, Prada N, Modi HN, Vasavada NB, Kim JS, Lee SH. Percutaneous endoscopic lumbar herniectomy for high-grade down-migrated L4-L5 disc through an $\mathrm{L}_{5}-\mathrm{S}_{1}$ interlaminar approach: A technical note. Minim Invasive Neurosurg 2010; 53:147-152.

18. Choi G, Lee SH, Lokhande P, Kong BJ, Shim CS, Jung B, Kim JS. Percutaneous endoscopic approach for highly migrated intracanal disc herniations by foraminoplastic technique using rigid working channel endoscope. Spine 2008; 33: $E_{508}-E_{515}$. 
19. Choi KC, Kim JS, Ryu KS, Kang BU, Ahn $\mathrm{Y}$, Lee SH. Percutaneous endoscopic lumbar discectomy for $\mathrm{L}_{5}$-S 1 disc herniation: Transforaminal versus interlaminar approach. Pain Physician 2013; 16:547-556.

20. Ruetten S, Komp M, Merk H, Godolias G. Full-endoscopic interlaminar and transforaminal lumbar discectomy versus conventional microsurgical technique: A prospective, randomized, controlled study. Spine (Phila Pa 1976) 2008; 33:931-939.

21. Knight M, Goswami A. Management of isthmic spondylolisthesis with posterolateral endoscopic foraminal decompression. Spine 2003; 28:573-581.

22. Kim HS, Park JY. Comparative assessment of different percutaneous endoscopic interlaminar lumbar discectomy (PEID) techniques. Pain Physician 2013; 16:359-367.

23. Kim CH, Chung CK. Endoscopic interlaminar lumbar discectomy with splitting of the ligament flavum under visual control. J Spinal Disord Tech 2012; 25:210-217.

24. Yeung AT, Tsou PM. Posterolateral endoscopic excision for lumbar disc herniation: Surgical technique, outcome, and complications in 307 consecutive cases. Spine (Phila Pa 1976) 2002; 27:722-731.

25. Schubert M, Hoogland T. Endoscopic transforaminal nucleotomy with foraminoplasty for lumbar disk herniation. Oper Orthop Traumatol 2005; 17:641-661.

26. A hn Y, Oh HK, Kim H, Lee SH, Lee HN.
Percutaneous endoscopic lumbar foraminotomy: an advanced surgical technique and clinical outcomes. Neurosurgery 2014; 75:124-133; discussion 132-133.

27. Yeung AT, Yeung CA. Advances in endoscopic disc and spine surgery: Foraminal approach. Surg Technol Int 2003; 11:255-263.

28. Lee S, Kim SK, Lee SH, Kim WJ, Choi WC, Choi G, Shin SW. Percutaneous endoscopic lumbar discectomy for migrated disc herniation: Classification of disc migration and surgical approaches. Eur Spine ] 2007; 16:431-437.

29. Shamji MF, Bains I, Yong E, Sutherland G, Hurlbert RJ. Treatment of herniated lumbar disk by sequestrectomy or conventional diskectomy. World Neurosurg 2013. [Epub ahead of print].

30. Fakouri B, Shetty NR, White TC. Is sequestrectomy a viable alternative to $\mathrm{mi}$ crodiscectomy? A systematic review of the literature. Clin Orthop Relat Res 2014 [Epub ahead of print].

31. Kotil K, Koksal NS, Kayaci S. Long term results of lumbar sequestrectomy versus aggressive microdiscectomy. J Clin $\mathrm{Neu}$ rosci 2014; 21:1714-1718.

32. Schick U, Elhabony R. Prospective comparative study of lumbar sequestrectomy and microdiscectomy. Minim Invasive Neurosurg 2009; 52:180-185.

33. Barth M, Weiss C, Thome C. Two-year outcome after lumbar microdiscectomy versus microscopic sequestrectomy: Part 1: Evaluation of clinical outcome. Spine (Phila Pa 1976) 2008; 33:265-272.
34. Barth M, Diepers M, Weiss C, Thome C. Two-year outcome after lumbar microdiscectomy versus microscopic sequestrectomy: Part 2: Radiographic evaluation and correlation with clinical outcome. Spine (Phila Pa 1976) 2008; 33:273-279.

35. Suess O, Brock M, Kombos T. Motor nerve root monitoring during percutaneous transforaminal endoscopic sequestrectomy under general anesthesia for intra- and extraforaminal lumbar disc herniation. Zentralbl Neurochir 2005; 66:190-201.

36. Lee $\mathrm{DH}$, Kim $\mathrm{NH}$, Park JB, Hwang $\mathrm{C}$, Lee CS, Kim YT, Kang SJ, Rhee JM. CT scan assessment of the pathway of the true lateral approach for transforaminal endoscopic lumbar discectomy: Is it possible? J Bone Joint Surg Br 2011; 93:1395-1399.

37. Jasper GP, Francisco GM, Telfeian AE. Endoscopic transforaminal discectomy for an extruded lumbar disc herniation. Pain Physician 2013; 16:E31-E35.

38. Jasper GP, Francisco GM, Telfeian AE. $A$ retrospective evaluation of the clinical success of transforaminal endoscopic discectomy with foraminotomy in geriatric patients. Pain Physician 2013; 16:225-229.

39. Bao MD, Gao BG, Wang YP, Wang GY. Therapeutic effects of microendoscopic discectomy (MED) for the treatment of lumbar disc herniation with a follow-up. Zhongguo Gu Shang 2008; 21:608-609. 
